\title{
Nursing Care of Critically Ill Patient Infected With COVID-19 Virus in China, Shanghai: A Case Report
}

\author{
Lin $Z^{1}$, Yan $G^{1,2 *}$, Ping $Y^{1}$, Yuanhao $W^{1}$, Jingjing $G^{1}$, Lin $W^{1}$, Jianliang $Z^{1}$, \\ Hongzhou $\mathrm{L}^{1}$ and Tongyu $\mathrm{Z}^{1}$ \\ ${ }^{1}$ Shanghai Public Health Clinical Center, China \\ ${ }^{2}$ School of nursing, Fudan University, China
}

*Corresponding author: GAO Yan, Shanghai Public Health Clinical Center, Fudan University, China, Email: 18211170010@fudan.edu.cn

\section{Case Report}

Volume 4 Special Issue 1

Received Date: April 22, 2020

Published Date: May 25, 2020

DOI: $10.23880 /$ nhij-16000S1-001

\section{Abstract}

COVID-19 has broken out in Wuhan and spread rapidly to most provinces and cities in China since December 2019. It has caused serious damage to the lives of patients and Chinese society and economy. In order to provide high-quality care for severe patients, reduce the incidence of nosocomial infection, and improve the cure rate, the nursing department of Shanghai Public Health Clinical Center proposed the nursing measures for the patient with severe pneumonia caused by COVID-19 virus infection according to the existing diagnosis and treatment and nursing guidance related to COVID-19.

Keywords: COVID-19; The Critically Ill Patient; Nurse; Case Report

Abbreviations: CRRT: Continuous Renal Replacement Therapy; ECMO: Extracorporeal Membrane Oxygenation; ACT: Activated Coagulation Time; Hct: Hematocrit; APTT: Activated Partial Thromboplastin Time.

\section{Introduction}

Coronavirus disease 2019(COVID-19) is a newly infectious disease caused by a newly discovered coronavirus. Most people infected with COVID-19 virus will develop mild to moderate respiratory illness that can be cured without special treatment. Older people, as well as those with underlying conditions such as cardiovascular disease, diabetes, chronic respiratory diseases, and cancer are more likely to develop serious illnesses [1]. Since December 2019, a number of cases of patients infected with COVID-19 virus have been found in Wuhan, China. With the spread of the epidemic, such cases have also been found in other parts of China and other countries [2]. Up to April 4, the global total number of patients confirmed COVID-19 cases climb above 1.05 million and the total number of deaths was 57,000 [3]. On January 20, 2020, the Chinese National Health Commission included the disease in the category B infectious diseases stipulated in the law of the people's Republic of China on the prevention and control of infectious diseases, and adopted the prevention and control measures for category A infectious diseases [4]. Shanghai Public Health Clinical Center (SPHCC) is a designated hospital for the admission and treatment of adult patients infected with COVID-19 virus in Shanghai during the COVID-19 epidemic. Up to March 25, there were 431 confirmed cases of COVID-19 diagnosed cases, 5 of which died, and 318 of which were discharged from hospital. The experience of care for a critically ill patient with COVID-19 is described below.

\section{Case Introduction}

The patient, a 65-year-old male, was quarantined because his wife was diagnosed as COVID-19. He developed white sputum and weakness on January 31 . The patient had no other symptoms such as aversion to cold, fever, headache, nasal obstruction, snivel, abdominal pain, diarrhea, chest tightness, shortness of breath, nausea and vomiting. The patient was admitted to Huashan hospital on January 31 for 
the COVID-19 nucleic acid test. After confirmed by the Chinese Centers for Disease Control and Prevention, the patient was admitted to the Shanghai Municipal Public Health Clinical Center after being diagnosed as COVID-19. The patient had a history of hypertension, diabetes and Alzheimer's.

On the day of admission, the nurses gave the patient routine emergency care, oxygen inhalation, soft diet, ECG monitoring. According to the doctor's advice, the nurse gave the patient Shuanghuanglian symptomatic treatment, gives hypoglycemic treatment due to his diabetes and monitored vital signs of the patient. On the night of February 2, the patient's chest tightness and shortness of breath became worse. The nurse gave the patient high flux inhalation of oxygen. Next morning, the patient's chest tightness and shortness of breath improved and his oxygen saturation reached $100 \%$. The patient developed symptoms of acute cardiac insufficiency, such as decreased urine output, rapid breathing, and increased heart rate on February 11. The patient was strictly controlled intake and output fluid volumes, given intermittent intravenous diuretic therapy, and continuously monitored for urine volume. On February 14 , the patient had cerebral infarction, the oxygenation index was lower than 100 , and the lactic acid was on the rise. In order to reduce breathing and improve oxygenation, the patient was given endotracheal intubation, ventilator assisted ventilation, and sedative and analgesic muscle relaxants. The patient was placed with a deep venous catheter and rated as critically ill patient. The patient's creatinine continued to rise and he developed anuria on February 17. The nurse treated him with bedside continuous renal replacement therapy (CRRT). After the patient turned over, the blood oxygen saturation dropped from about $99 \%$ to less than $80 \%$ on February 18. The effect of lung recruitment was not good. The extracorporeal Membrane Oxygenation (ECMO) treatment was initiated to improve the oxygen supply and oxygen consumption of the patient. Besides, in order to maintain the stability of the patient's internal environment, CRRT was combined. The ECMO was removed on March 26. The patient changed to high flow oxygen inhalation and enhanced rehabilitation exercise on March 27. The patient's limb hemiplegia caused by cerebral infarction was improved, and the renal failure was improved, so he was transferred to the department of infection and immunology for further treatment of cerebral infarction and renal failure. The patient's blood pressure, blood sugar, and intravenous fluids continued to be controlled. The nurse provided the patient with gastrointestinal nutrition support, tracheal incision and tracheal catheter care. On April 18, 2020, the patient underwent sitting balance training, oral ice stimulation training, tongue muscle training, swallowing training and breathing training with the assistance of a rehabilitation therapist.

\section{Nursing Assessment}

(1) The degree of hypoxia of the patient, such as whether the patient has any disturbance of consciousness like restlessness, and whether the skin mucosa color is cyanotic are assessed. (2) whether the patient's water intake and outlet are balanced should be assessed. (3)the nutriture of the patient should be assessed. (4)The patient's awareness of the disease and psychological state should be assessed by nurses.

\section{Condition Observation}

(1) Observe the vital signs and peripheral blood oxygen saturation of the patient. (2) Observe the patient's cough and expectoration. (3) Record the intake and output of liquid, and monitor the renal function, electrolyte and blood gas analysis.

\section{General Care}

(1) Nurses implement oxygen therapy and observed the treatment effect based on the doctor's prescriptions. The oxygen therapy device is dedicated to prevent crossinfection; (2)The nurse conducts 24-hour continuous ECG monitoring for the patient. The heart rate, respiratory rate, blood pressure and blood oxygen saturation of the patient are measured every hour, and the body temperature is measured every 4 hours. (3)The nurse should establish venous access reasonably, follow the doctor's instructions to control the infusion rate, use an infusion pump if necessary. (4) For the critically ill patient, catheters should be retained. Nurses have to calculate 24-hour daily liquid intake and output of the patient. (5)All kinds of catheters should be unobstructed and properly fixed. (6) For the patient without special posture requirements, the head of the bed needs to be raised to $30^{\circ}$. (7)Nurses have to keep the bed unit clean and prevent the pressure injury of skin. Besides, records of intensive care have to be finished in time.

\section{Nursing of ECMO}

World Health Organization recommends that ECMO (Figure 1) technology can be used for patients with acute respiratory distress syndrome caused by COVID-19 virus infection [5]. The main principle of ECMO is to draw venous blood out of the body through an intravenous catheter, and then the venous blood passes through an external oxygenator to carry out oxygen and remove carbon dioxide. The oxygenated blood is then returned to the body through the vein or artery. It can replace the cardiopulmonary function of the patient partly or completely for a long period of time, maintain the blood oxygen supply of various organs of the body, and provide short or long-term cardiopulmonary support. 
1 In the implementation of ECMO nursing, an ECMO rescue team needs to be set up at first. The group consists of the department director (person in charge), the systemic circulation group, the ICU attending doctor (coordinator) and two ICU nurses. The main responsibilities of the person in charge are to organize and formulate the clinical treatment and scientific research development direction of ECMO, and determine the responsibilities of the ECMO team members. The coordinator is mainly responsible for the daily affairs of the ECMO team, including coordinating the internal and external contacts of the team, quality control and monitoring of ECMO treatment, formulating rules, specifications and operating procedures of ECMO treatment, collecting ECMO case data (ECMO patient data, follow-up, etc.), personnel dispatching, equipment maintenance and consumables management, and provide the necessary support and services for team members. Team members must keep their mobile phones on for $24 \mathrm{~h}$. The care of ECMO the patient is led by 2 professional ICU nurses, who closely observe and evaluate the patient's condition, cooperate with the doctor for treatment, and assist in monitoring abnormal conditions during the auxiliary circulation.

\section{Key Implementation Points}

\section{Evaluation}

(1)Environment: The environment is safe and meets the requirements of ECMO. (2)Patient Assessment: Patient's vital signs, blood oxygen saturation and skin condition of neck and groin.

\section{Observation}

(1)patient awareness; (2)ECG, blood pressure and mean arterial pressure; (3)central venous pressure(CVP); (4)activation of thrombin time (ACT); (5)blood oxygen saturation; (6)electrolytes and urine output.

\section{Preparation of Materials}

(1)ECMO machine, including extracorporeal circulation package $\times 1$, centrifugal pump head, extracorporeal circulation artery filter, ECMO water tank, blood oxygen saturation and hematocrit measurement system; (2)disinfection package, including abdominal dressing package, venous incision bag, suture bag, deep vein puncture bag, sterile toothless vascular clamp $\times 2$; (3)other preparations including BS blood oxygen saturation sensor, pressurized bag, invasive blood pressure monitor, catheter (arteriovenous insertion tube $\times 2$, arterial tube $\times 2$ ), disposable medicine bowl, iodophor, gauze, vascular forceps $\times 1$, syringes, anal sterile adhesive film $\times 2$, large transparent film $\times 4$, large straight forceps $\times 6$ (the front end is protected with tourniquet), roll of elastic tape $\times 1$; (4)drug preparation, including 3 bags of lactated Ringer's solution, 2 bottles of human blood albumin, heparins $\times 2,1$ bag of $500 \mathrm{ml}$ saline, 1 bottle of sterilized water for injection $(500 \mathrm{ml})$.

\section{Operation Points}

(1)Check bed number and name; (2)Machine preparation: Inject lactated Ringer's solution and human blood albumin into the centrifugal pump and exhaust the air; (3)Take the prostrate position for the patient, establish invasive blood pressure monitoring, expose the skin, disinfect the skin, and lay the sterile area; (4)Assist doctors to choose the same side of the internal jugular vein and inguinal vein for puncture, insert an arteriovenous cannula; (5) Use sterile toothless vascular forceps to clamp the catheter, and connect the catheter on the artificial lung machine; 6) Fixed catheter (An iodine film-large film-gauze compression hemostasiselastic tape); (7) Observe the patient's vital signs, blood oxygen saturation, puncture site bleeding and parameters of the ECMO machine; 8Clean up the objects, and send the equipment package to the supply room for disinfection; (9)Wash hands and keep records.

\section{Nursing Routine}

During the treatment of ECMO, the patient should be given sufficient sedation and analgesia. The catheter should be properly fixed. Nurses have to keep the ECMO catheter unobstructed and observe the speed and flow rate of the centrifugal pump. The flow rate should be kept constant. Nurses observe whether there is leakage at the outlet of membrane oxygenator and whether there is any vibration of the vein catheter. If there is any abnormality, inform the doctor in time. Besides, medical workers have to ensure the continuous uninterrupted oxygen supply of the membrane oxygenator, observe whether there is active bleeding, bleeding, swelling at the puncture point of the ECMO of the patient, change the dressing in time, and maintain a sterile environment. Venous oxygen saturation $\left(\mathrm{SvO}_{2}\right)$, mean arterial pressure (MAP), $\mathrm{PaO}_{2}, \mathrm{PaCO}_{2}$, arterial blood gas analysis, activated coagulation time (ACT), hematocrit (Hct), urine volume and body temperature of patient should be monitored. If there is an S-G catheter inserted, cardiac output and pulmonary artery pressure should be monitored. For the prevention and care of bleeding, the nurse should monitor the patient's platelet count, activated partial thromboplastin time (APTT) and other indicators related to coagulation function. The nurse should give the patient anticoagulant treatment according to the doctor's instructions. If necessary, the blood products should be transfused. The nursing operations should be gentle to avoid bleeding. For the prevention and care of hemolysis, the nurse monitors the patient's plasma free hemoglobin concentration; urine volume and urine color, and informs the doctor if there 
is any abnormality. For nutritional support, the enteral nutrient solution should be used for the patient with ECMO. The color and nature of gastric juice and gastrointestinal motility, exhaust, and defecation should be monitored to prevent flatulence. Parenteral nutrition can be used at the appropriate time.

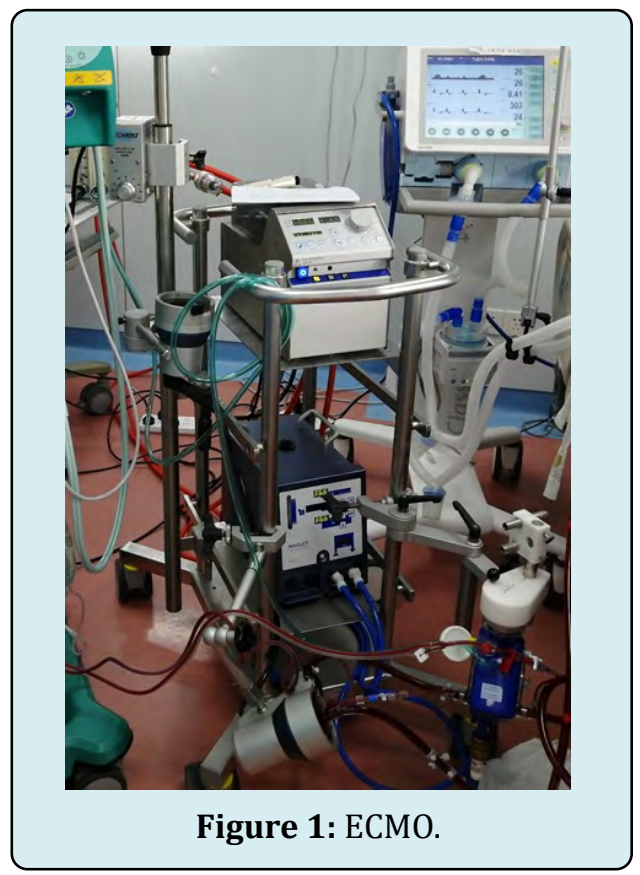

\section{Nursing of CRRT}

CRRT (Figure 2) is an important development of blood purification treatment technology which can remove a variety of metabolites, poisons, drugs and other pathogenic biomolecules while regulating body fluid balance through a series of new technologies such as ultrafiltration, perfusion and adsorption [6,7]. Receiving continuous renal replacement therapy is one of the important means to treat the patient with COVID-19 virus infection. The Diagnosis and Treatment Program of COVID-19(Version 5) clearly states that the use of extracorporeal blood purification technology may be considered for the critically ill patient with high inflammatory response if possible [8].

\section{Basic Nursing}

The environment in the ward should be comfortable, clean and warm. The table and the floor should be wiped with chlorine-containing disinfectant. Nurses have to perform sterile operation strictly during nursing operation, disinfect the catheter and surrounding skin every day. In addition, during care, the nurse has to observe the catheter of the patient to avoid fall-off, discount, and blockage of the catheter.

\section{Nursing and Observation of the Catheter}

During the treatment of CRRT, the patient's consciousness, vital signs, urine volume, body temperature and other vital signs should be closely observed and recorded in detail. Since the duration of CRRT is often very long, even flushing and fluid replacement treatments are required, so in order to avoid bacterial contamination, heparin should be used to seal the tube to keep the interface clean. The catheter should be properly fixed during CRRT.

\section{Water and Electrolyte Balance Care}

During CRRT treatment, the nurse must monitor the patient's water and electrolyte and monitor blood gas analysis every 4 hours, and renal function and electrolytes 2 to 3 times in one day [9]. In addition, the nurse supplements calcium and adjusts CRRT according to the patient's condition. The nurse calculates the fluid intake and output of the patient every hour, and adjusts the flow rate according to the patient's condition and blood pressure to ensure the best treatment effect.

\section{Prevention and Nursing of Bleeding}

Since heparin is used in the treatment of CRRT, which can lead to the tendency of bleeding in the patient, so it is it is necessary to observe the bleeding in the wound and upper digestive tract. If the patient has bleeding, protamine can be injected to neutralize heparin. In addition, in order to reduce bleeding, the application dose of heparin can be appropriately reduced according to the doctor's order.

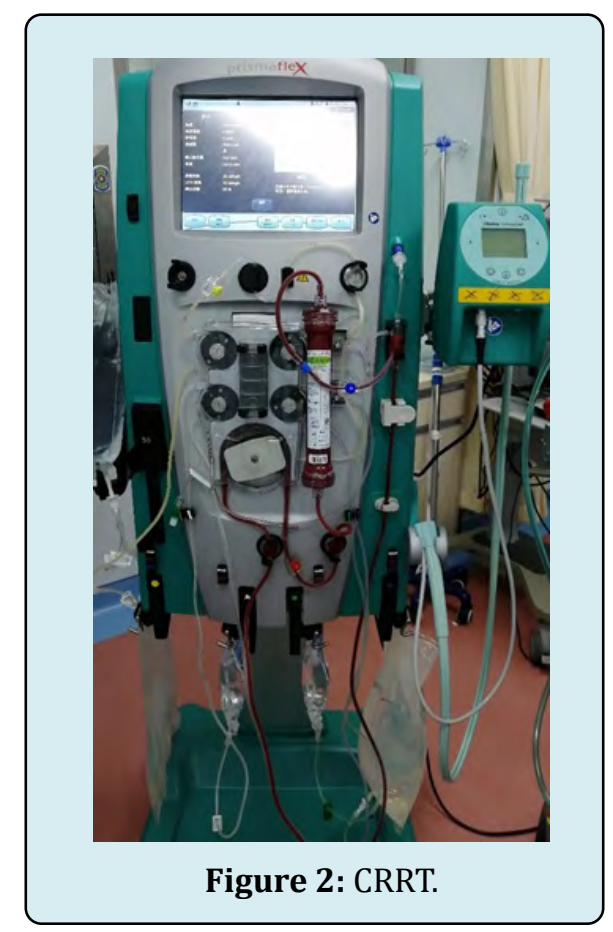




\section{Respiratory Function Exercise for the Patient with COVID-19}

For this patient with a stable convalescence, he still needs to stay in bed. So it is recommended to carry out bed rehabilitation training to reduce the complications that may be caused by staying in bed for a long time, improve the cardiopulmonary function and physical and mental state of the patient, promote the recovery of pneumonia and improve the prognosis [10].

\section{Abdominal Breath Training}

The patient takes the supine or semi-recumbent position, with one hand is placed on the chest and the other hand is placed on the abdomen to feel the ups and downs of breathing. The patient inhaled slowly through the nose, keeping the shoulders and chest relaxed while inhaling, and only the abdomen heaved with the inhalation. The patient slowly exhales through the mouth while the abdomen retracts. The ratio of inhalation and exhalation is $1: 2$. The patient is trained $5 \sim 7$ times for $5 \sim 15$ minutes.

\section{Lip Contract Breath Training}

The patient takes supine or semi-recumbent position, inhales through the nose while keeping the patient's mouth closed. Then the patient exhales slowly through the mouth (in a whistle-like), and contracts the abdomen at the same time. The ratio of inhalation to exhalation time is $1: 2$ or $1: 3$. The patient is trained 7 8 times for 10 20 minutes.

\section{Conclusion}

The patient was diagnosed with COVID-19. The disease progressed rapidly. The infection was severe. The treatment and care was extremely difficult, and the health care workers were at great risk of exposure. At present, the application of ECMO technology is of great significance in maintaining hemodynamics stability, alleviating ischemia and anoxia, restoring cardiopulmonary function and prolonging life of the patient. The application of ECMO in the treatment of the patient with COVID-19 requires multi-disciplinary cooperation, meticulous observation, monitoring and effective nursing cooperation. In the process of operation, it is necessary to closely monitor the indexes of ECMO and the patient's vital signs, standardize the nursing measures such as drug management, catheter management and protection against hospital infection, and actively prevent and deal with complications so as to improve the success rate of rescuing the patient.

\section{References}

1. WHO (2005) Coronavirus, World Health Organization.

2. WHO (2020) Pneumonia of unknown cause - China, World Health Organization.

3. WHO (2020) Coronavirus disease 2019 (COVID-19) Situation Report-75, World Health Organization.

4. CCDCP (2020) Announcement by the national health commission, Chinese Centers for Disease Control and Prevention.

5. WHO (2020) Clinical management of severe acute respiratory infection when novel coronavirus (2019$\mathrm{nCoV}$ ) infection is suspected: interim guidance, World Health Organisation, pp: 1-10.

6. Mencía S, López M, López-Herce J, Ferrero L, RodríguezNúñezA (2014) Simulating continuous renal replacement therapy: usefulness of a new simulator device. J Artif Organs 17(1): 114-117.

7. Shi S, Jia S, Liu J, Chen G, He S (2014) Continuous renal replacement therapy as a supportive treatment for acute pediatric necrotizing fasciitis. Cell Biochem Biophys 69(2): 219-223.

8. NHI (2020) Diagnosis and treatment of pneumonia caused by COVID-19 infection (Version 5), National Health Commission.

9. Zhan WJ (2010) Nursing of critically ill patients with continuous renal replacement therapy. Chinese Journal of Practical Nursing 26(27): 23-24.

10. Liu XD, Liu L, Lu YF (2020) Guidance and suggestions on rehabilitation training of integrated traditional Chinese and western medicine for functional recovery of patients with COVID-19. Shanghai Journal of Traditional Chinese Medicine 54(3): 9-13. 